\title{
Prevalence and outcome of dysnatremia in patients with COVID-19 compared to controls
}

\author{
Cihan Atila1,2,*, Clara O Sailer(101,2,*, Stefano Bassetti ${ }^{4}$, Sarah Tschudin-Sutter ${ }^{5,2}$, Roland Bingisser ${ }^{6}$, \\ Martin Siegemund ${ }^{7}$, Stefan Osswald ${ }^{3}$, Katharina Rentsch ${ }^{8}$, Marco Rueegg ${ }^{10}$, Sabrina Schaerli6, \\ Gabriela M Kuster ${ }^{3}$, Raphael Twerenbold ${ }^{3}$ and Mirjam Christ-Crain ${ }^{1,2}$
}

1Department of Endocrinology, Diabetology and Metabolism, University Hospital Basel, Basel, Switzerland, ${ }^{2}$ University of Basel, Department of Clinical Research, Basel, Switzerland, ${ }^{3}$ Department of Cardiology, University Hospital Basel, Basel, Switzerland, ${ }^{4}$ Department of Internal Medicine, University Hospital Basel, Basel, Switzerland, ${ }^{5}$ Division of Infectious Disease \& Hospital Epidemiology, University Hospital Basel, Basel, Switzerland, ${ }^{6}$ Emergency Department, University Hospital Basel, Basel, Switzerland, 'Department of Intensive Care, University Hospital Basel, Basel, Switzerland, and ${ }^{8}$ Department of Laboratory Medicine, University Hospital Basel, Basel, Switzerland

${ }^{*} \mathrm{C}$ Atila and C O Sailer contributed equally to this research and are joint first authors

Correspondence should be addressed to R Twerenbold or M Christ-Crain

Email

raphael.twerenbold@usb.ch or mirjam.christ-crain@usb. $\mathrm{ch}$

\begin{abstract}
Objective: The pandemic of coronavirus disease (COVID-19) has rapidly spread globally and infected millions of people. The prevalence and prognostic impact of dysnatremia in COVID-19 is inconclusive. Therefore, we investigated the prevalence and outcome of dysnatremia in COVID-19.

Design: The prospective, observational, cohort study included consecutive patients with clinical suspicion of COVID-19 triaged to a Swiss Emergency Department between March and July 2020.

Methods: Collected data included clinical, laboratory and disease severity scoring parameters on admission. COVID-19 cases were identified based on a positive nasopharyngeal swab test for SARS-CoV-2, patients with a negative swab test served as controls. The primary analysis was to assess the prognostic impact of dysnatremia on 30-day mortality using a cox proportional hazard model.

Results: 172 (17\%) cases with COVID-19 and 849 (83\%) controls were included. Patients with COVID-19 showed a higher prevalence of hyponatremia compared to controls $(28.1 \%$ vs $17.5 \%, P<0.001)$; while comparable for hypernatremia ( $2.9 \%$ vs $2.1 \%, P=0.34)$. In COVID-19 but not in controls, hyponatremia was associated with a higher 30 -day mortality (HR: 1.4, 95\% Cl: 1.10-16.62, $P=0.05$ ). In both groups, hypernatremia on admission was associated with higher 30-day mortality (COVID-19 - HR: 11.5, 95\% Cl: 5.00-26.43, P<0.001; controls - HR: 5.3, 95\% Cl: 1.60-17.64, $P=0.006)$. In both groups, hyponatremia and hypernatremia were significantly associated with adverse outcome, for example, intensive care unit admission, longer hospitalization and mechanical ventilation.

Conclusion: Our results underline the importance of dysnatremia as predictive marker in COVID-19. Treating physicians should be aware of appropriate treatment measures to be taken for patients with COVID-19 and dysnatremia.
\end{abstract}

\section{Introduction}

The ongoing pandemic of coronavirus disease 2019 (COVID-19), caused by severe acute respiratory syndromecoronavirus-2 (SARS-CoV-2), has rapidly spread worldwide and infected millions of people with increasing numbers of infection rate and deaths (World Health Organization). SARS-CoV-2 is characterized by clinical symptoms such as fever $(83.82 \%)$, cough $(81.8 \%)$, fatigue $(69.6 \%)$, and shortness of breath (31\%) (1). Data from large cohort https://eje.bioscientifica.com https://doi.org/10.1530/EJE-20-1374 (c) 2021 European Society of Endocrinology Printed in Great Britain
Published by Bioscientifica Ltd. 
studies have shown that physician diagnosed pneumonia rate of up to $91 \%$ in hospitalized patients with COVID-19 $(2,3)$.

In patients with pneumonia, dysnatremia is common. In fact, hyponatremia has been described with an incidence rate of up to $30 \%$ in hospitalized patients (4, $5,6,7,8)$, while hypernatremia is less frequent with an incidence rate of $3 \%$ in hospitalized patients $(9,10,11$, $12,13)$. The mechanisms causing dysnatremia in patients with pneumonia are not well-understood and include decreased water clearance, increased saliuretic therapy and an increased rate of the syndrome of inappropriate antidiuresis (SIAD) $(7,8,14,15)$.

The plasma sodium level in patients with pneumonia plays an important role for severity assessment and is therefore used in scores like the pneumonia severity index (PSI) (15). Many studies have shown the association between dysnatremia, especially hyponatremia and worse outcome parameters like mortality and length of hospital stay $(19,20)$. Large cohort studies have reported an association between hyponatremia and severity parameters such as higher rates of intensive care unit (ICU) admission, longer ICU stay and mechanical ventilation as an important prognostic marker in patients with pneumonia (16). A meta-analysis with 13816 patients showed a significant association of hyponatremia improvement with reduction of overall mortality up to $60 \%$ compared to patients without hyponatremia improvement $(11,17)$.

To date, the prevalence and outcome of dysnatremia in patients tested positive for COVID-19 remains inconclusive. While most studies on electrolyte disturbances in patients with COVID-19 have shown overall significantly lower sodium blood concentrations, other studies in COVID-19 reported hypernatremia to be more frequent $(2,18)$, but no study reported comparison to a control group.

The aim of our study was to investigate the prevalence of dysnatremia and the impact on patient-outcome, that is, mortality rate and rehospitalization, and severity of disease in COVID-19. We hypothesized an increased rate of dysnatremia in patients with COVID-19 compared to controls and an association with increased mortality and rehospitalization. Furthermore, we hypothesized that the previously observed association of dysnatremia with clinical severity parameters is also true for patients with COVID-19.

\section{Methods}

\section{Study design, population and inclusion criteria}

The prospective, observational, cohort study included consecutively enrolled patients aged 18 years and older presenting with clinical suspected or confirmed SARSCoV-2 infection to the emergency department (ED) of the University Hospital in Basel, Switzerland, during the first wave of COVID-19 pandemic between March 2020 and July 2020. All patients underwent nasopharyngeal SARS-CoV-2 PCR swab tests. Patients were considered COVID-19 positive, in the following referred to as cases, if at least one SARS-CoV-2 PCR swab test performed at day of ED presentation or within 14 days prior to or post ED presentation was positive in combination with clinical signs and symptoms of COVID-19 infection. Patients with negative SARS-CoV-2 PCR swab test were considered controls. All participating patients or their legally authorized representatives consented by signing a local general consent form. The study was registered on ClinicalTrials.gov (ClinicalTrials.gov NCT04366765), conducted according to the principles of the Declaration of Helsinki, and approved by the Ethical Committee Northwest and Central Switzerland. The authors designed the studies, gathered, and analyzed the data according to the STROBE guidelines (19), vouched for the data and analysis, wrote the paper, and decided to submit it for publication.

The total number of included patients $(n=1041)$ was grouped into patients with confirmed COVID-19 ( $n=172$; cases) and patients without COVID-19 but similar symptoms on admission ( $n=849$; controls). Twenty patients were excluded for absence of sodium levels measured on admission (Fig. 1). In case of multiple presentations to the $\mathrm{ED}$ during the study duration, we only considered the index presentation. According to the sodium levels on admission, patients were sub-grouped in normonatremic (135-145 mmol/L), hyponatremic $(<135$ $\mathrm{mmol} / \mathrm{L})$, and hypernatremic (>145 $\mathrm{mmol} / \mathrm{L})$.

\section{Follow-up}

Patients were contacted over telephone calls or in written form 30 days after discharge by research physicians or study nurses. Information about current health, rehospitalizations, and adverse events were obtained, guided by a predefined set of questions and item-checklists. 


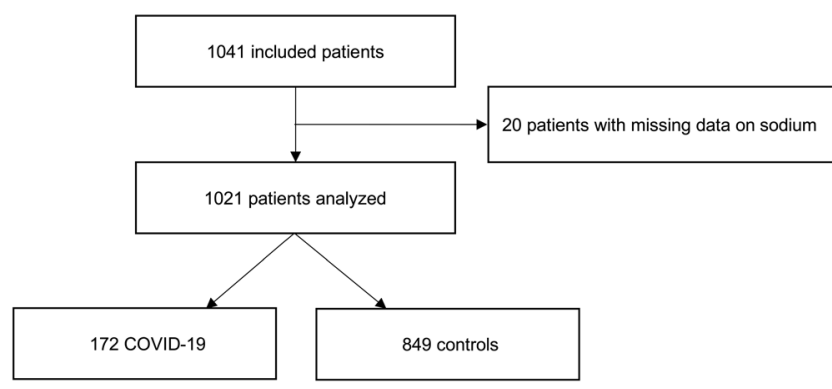

Figure 1

Study flowchart. Flowchart showing patient enrollment. In total, 1041 patients were included in the study. Altogether 1021 patients were analyzed; 20 patients were excluded for absence of sodium levels measured on admission.

Records of hospitals and primary care physicians, as well as national death registries, were queried for additional information, if applicable.

\section{Clinical assessment}

All patients underwent a thorough clinical assessment by the treating ED physician according to local standard operating procedure. Vital parameters including body temperature, respiratory rate, oxygen saturation, heart rate, and blood pressure were assessed in every patient. The patients' management was left to the discretion of the attending physicians and in accordance with local standard operating procedures.

\section{Blood sampling}

Blood samples were routinely drawn in every patient (both cases and controls) at time of ED presentation. Routine laboratory parameters, for example, sodium and c-reactive protein (CRP), were determined in every patient as part of the local standard operating procedure in COVID-19 suspects. Timing and type of subsequent laboratory measurements during hospital stay were left to the discretion of the treating physicians and were not part of this study protocol.

\section{Study outcomes}

The primary outcome was to explore the association of admission dysnatremia, that is, hyponatremia and hypernatremia, with 30-day mortality and 30-days rehospitalization in patients with confirmed COVID-19 as compared to controls.
Secondary outcomes were to assess the association of admission dysnatremia with adverse outcome such as length of hospital stay, need for ICU admission, and need for invasive mechanical ventilation in COVID-19 compared to controls. Furthermore, we investigated the association of dysnatremia on admission with clinical severity parameter, measured by vital parameters such as body temperature, respiratory rate, oxygen saturation, and National Early Warning Score (NEWS) (20) on admission in both groups, and with clinical symptoms, such as fever and shivering.

\section{Statistical analysis}

Baseline variables of continues data are shown as median with interquartile range (IQR) and categorical variables as number $(n)$ with percentage $(\%)$, respectively.

The primary endpoint was the association of hyponatremia and hypernatremia with time to 30-day mortality using a Cox proportional hazard model for patients with COVID-19 and controls. In a multivariable Cox proportional hazard models, we adjusted for age, sex, and number of comorbidities. The same method was used to assess the association of sodium levels on admission with time to 30-day rehospitalization rate in both groups. Graphical time to event analyses was performed using the Kaplan-Meier method.

Secondary endpoints were analyzed using univariable and multivariable logistic regression models to assess the association of serum sodium levels, that is, subgroups hyponatremia, normonatremia and hypernatremia, on adverse outcome, for example, length of hospital stay, ICU admission, and need for mechanical ventilation. Furthermore, we used the same method to assess the association of sodium levels parameters presenting severity of disease, for example, body temperature, respiratory rate, oxygen saturation, and NEWS on admission, and on symptoms, for example, fever and shivering. In the multivariable regression model, we adjusted for further known variables influencing sodium levels such as sex, age and the presence of comorbidities. The sum in numbers of predefined comorbidities per patient was used for the adjustment, ranging from 0 to 12 . Comorbidities included presence of coronary heart disease, heart failure, arterial hypertension, pneumopathy, renal failure, hepatopathy, obesity $\left(\right.$ BMI $>25 \mathrm{~kg} / \mathrm{m}^{2}$ ), rheumatological disease, immunosuppression inclusive human immunodeficiency virus (HIV) infection, cerebrovascular disease, active neoplastic disease, and diabetes mellitus. The independent variables were included in a stepwise way. 
Data were analyzed using $R$ statistical software (21). A two-sided $P$-value of $<0.05$ was considered to be statistically significant.

\section{Results}

\section{Baseline characteristics}

In the present analysis, 1021 patients were included, of whom 172 (17\%) had a positive and 849 (83\%) had a negative nasopharyngeal swab for SARS-CoV-2, with an overall median age of 59 years (IQR: 42-73), 45\% females. Overall, the median length of hospitalization was 7 days (IQR: 4.5-13.5) in patients with COVID-19 and 6 days (IQR: 4-10) in controls. Baseline characteristics including comorbidities, medications and laboratory parameters, for example, CRP, of the subgroups are shown in Table 1.

\section{Prevalence of dysnatremia}

On admission, patients with COVID-19 as compared to controls showed a higher prevalence of hyponatremia ( $n=50,29.1 \%$ vs $n=149,17.6 \%$, OR 1.95, 95\% CI 1.33-2.83, $P<0.001)$. In contrast, hypernatremia was comparably prevalent in both groups on admission $(n=5$, $2.9 \%$ vs $n=18,2.1 \%, P=0.34$ ) (Fig. 2, Table 1 ).

\section{Association of admission dysnatremia with 30-day mortality and 30-day rehospitalization}

Follow-up data for in-hospital events were available for all patients while follow-up data 30 days after discharge were available for 1015 of 1021 patients. Overall, 44 of 1015 patients died during the first 30 days after admission, with $7.0 \%$ (12 of 171 patients) significantly more often in COVID-19 as compared to 3.8\% (32 of 844 patients) in controls (HR: 2.5, 95\% CI: 1.24-4.89, $P=0.009$ ). The leading cause of death was respiratory failure, followed by cardiovascular arrest, multi-organ failure, and septic shock.

In COVID-19, hyponatremia on admission in comparison to normonatremia showed a significant association with 30-day mortality (HR: 1.4, 95\% CI: 1.10 $16.62, P=0.05)$, whereas no association in controls was observed (HR: 0.93, 95\% CI: 0.36-2.46, $P=0.89$ ).

In both groups, hypernatremia on admission showed a higher 30-day mortality in comparison to normonatremia (COVID-19 - HR: 11.5, 95\% CI: 5.00-26.43, $P<0.001$; controls - HR: 5.3, 95\% CI: 1.60-17.64, $P=0.006$ ). Figure 3A, B and C show Kaplan-Meier survival curves for

Table 1 Baseline characteristics of both groups. Data from 1021 patients; 849 controls and 172 with positive nasopharyngeal swab test for SARS-CoV-2 in each subgroup (normonatremia, hyponatremia, and hypernatremia on admission). Data are presented as $n$ (\%) and median (IQR: 25th -75th percentile).

\begin{tabular}{|c|c|c|c|c|c|c|}
\hline & \multicolumn{3}{|c|}{ COVID-19 } & \multicolumn{3}{|c|}{ Controls } \\
\hline & Normonatremia & Hyponatremia & Hypernatremia & Normonatremia & Hyponatremia & Hypernatremia \\
\hline \multicolumn{7}{|l|}{ Patients } \\
\hline$n$ & 117 & 50 & 5 & 682 & 149 & 18 \\
\hline Age (years) & $54(41-65)$ & $63(52-75)$ & $70(65-71)$ & $57(38-74)$ & $67(54-76)$ & $71(57-76)$ \\
\hline Females & $55(47)$ & $18(36)$ & $3(60)$ & $290(43)$ & $62(42)$ & $9(50)$ \\
\hline \multicolumn{7}{|l|}{ Comorbidities } \\
\hline $\begin{array}{l}\text { Number of } \\
\text { comorbidities }\end{array}$ & $1.7(1.7)$ & $2.4(2.0)$ & $3.8(1.8)$ & 1.9 (1.9) & $2.4(1.8)$ & $3.3(2.8)$ \\
\hline Diabetes mellitus & $18(16)$ & $14(28)$ & $2(40)$ & $87(13)$ & $34(23)$ & $6(33)$ \\
\hline Hypertension & $45(39)$ & $25(50)$ & $4(80)$ & $272(40)$ & $66(44)$ & $9(50)$ \\
\hline Obesity & $41(35)$ & $21(42)$ & $4(80)$ & $216(32)$ & $45(30)$ & $5(28)$ \\
\hline \multicolumn{7}{|l|}{ Medication on admission } \\
\hline Diuretics & $20(17)$ & $9(18)$ & $3(60)$ & $139(21)$ & $28(19)$ & $8(44)$ \\
\hline ACE-inhibitors & $11(9)$ & $8(16)$ & $2(40)$ & $71(10)$ & $21(14)$ & $1(6)$ \\
\hline AT2-Blocker & $21(18)$ & $11(22)$ & $0(0)$ & $106(16)$ & $18(12)$ & $4(22)$ \\
\hline Analgesics & $33(28)$ & $11(22)$ & $4(80)$ & $143(21)$ & $35(23)$ & $5(28)$ \\
\hline B-blockers & $13(11)$ & $8(16)$ & $1(20)$ & $131(19)$ & $36(24)$ & $5(28)$ \\
\hline Steroids & $1(1)$ & $2(4)$ & $1(20)$ & $35(5)$ & $11(7)$ & $2(11)$ \\
\hline Immunosuppressants & $1(1)$ & $1(2)$ & $1(20)$ & $27(4)$ & $12(8)$ & $2(11)$ \\
\hline \multicolumn{7}{|l|}{$\begin{array}{l}\text { Laboratory values on } \\
\text { admission }\end{array}$} \\
\hline Sodium (mmol/L) & $138(136-141)$ & $132(130-134)$ & $148(148-155)$ & $139(137-141)$ & $132(129-133)$ & $147(146-151)$ \\
\hline Potassium (mmol/L) & $3.9(3.7-4.1)$ & $4.0(3.6-4.4)$ & $4.7(4.5-5.0)$ & $4.0(3.8-4.3)$ & $4.0(3.7-4.3)$ & $4.0(3.8-4.3)$ \\
\hline CRP (mmol/L) & $11.6(1.5-52.5)$ & $49.4(27.7-107.0)$ & $127.0(106.5-138.0)$ & $4.8(0.9-31.4)$ & $56.1(12.2-132.3)$ & $5.3(2.6-25.5)$ \\
\hline
\end{tabular}

$\mathrm{ACE}$, angiotensin converting enzyme; AT2, angiotensin 2; CRP, c-reactive protein; Obesity (BMI > 25 kg/m²); IQR, inter quartile range. 


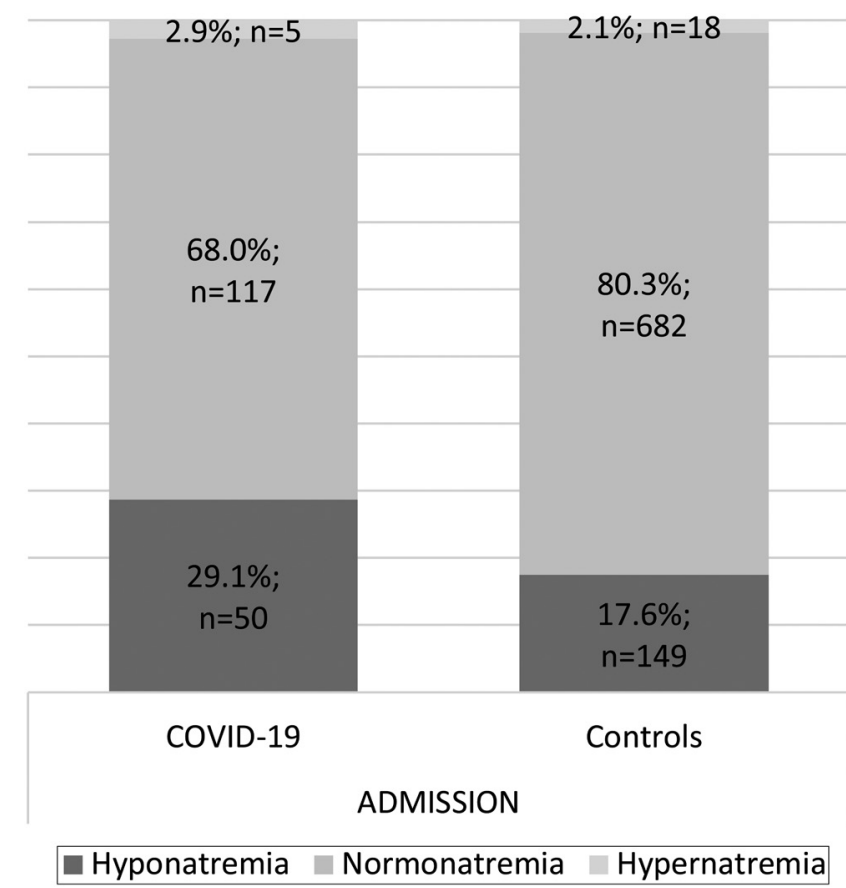

Figure 2

Prevalence of dysnatremia on admission in COVID-19 and controls. Sodium levels on admission in patients with COVID$19(n=172)$ and controls $(n=849)$ in each subgroup (hyponatremia, normonatremia, and hypernatremia). Data are presented as numbers $(n)$ and percentage of patients.

admission sodium and all-cause mortality for all patients, patients with COVID-19, and controls within the first 30 days after admission.

Overall, the 30-day rehospitalization rate in survivors was $6.0 \%$ ( $n=59$ of 982 patients); $5.0 \%$ ( $n=8$ of 159 ) in patients with COVID-19 and $6.2 \%(n=51$ of 823$)$ in controls (HR: $0.76,95 \%$ CI: 0.36-1.62, $P=0.48$ ). In an adjusted multivariable analysis, in both groups, hyponatremia as compared to normonatremia on admission showed no association to a higher 30-day rehospitalization rate (COVID-19- HR: 0.7, 95\% CI: 0.15-3.88, $P=0.74$; controls- HR: $1.7,95 \%$ CI: 0.93-3.16, $P=0.08$ ), which was similar for hypernatremia on admission as compared to normonatremia (COVID-19- HR: 0.7, 95\% CI: 0.33-1.23, $P=0.89$; controls- HR: $1.8,95 \%$ CI: $0.40-7.86, P=0.46$ ).

\section{Association of admission sodium levels with adverse outcome, severity parameters, and clinical symptoms}

After adjusting for age, sex, and comorbidities, irrespective of the COVID-19 status, hyponatremia and hypernatremia were associated with adverse outcome. Specifically, patients with hyponatremia and hypernatremia as compared to normonatremia showed a significant longer hospital stay (hyponatremia- OR: 2.7, 95\% CI: 2.59-2.93, $P<0.001$; hypernatremia- OR: 2.5, 95\% CI: 2.13-2.89, $P<0.001$ ), were significantly associated with higher ICU admission rates (hyponatremia- OR: 2.8, 95\% CI: 1.64-4.88, $P<0.001$; hypernatremia- OR: 10.3 , 95\% CI: 3.54-27.42, $P<0.001$ ), and were significantly linked to invasive mechanical ventilation (hyponatremia- OR: 3.3, 95\% CI: 1.67-6.63, $P<0.001$, hypernatremia- OR: 6.1, 95\% CI: 1.28-22.00, $P=0.01$ ) (Table 2).

Irrespective of the COVID-19 status, hyponatremia and hypernatremia on admission were associated with severity parameters. In detail, hyponatremia and hypernatremia as compared to normonatremia were associated with a higher respiratory rate on admission (hyponatremia- OR: 1.4, 95\% CI: 1.34-1.44, $P<0.001$; hypernatremia- OR: 1.2 95\% CI: $1.10-1.29, P=0.001$ ), and were associated with significantly lower oxygen levels $\left(\mathrm{SpO}_{2} \leq 93 \%\right)$ compared to normonatremic patients (hyponatremia- OR: $1.8,95 \% \mathrm{CI}: 1.16-2.68, P=0.006$; hypernatremia- OR: $2.9,95 \% \mathrm{CI}: 1.19-5.98, P=0.009$ ), and showed a strong association with a higher NEWS on admission (hyponatremia- OR: 1.3, 95\% CI: 1.23-1.47, $P<0.001$; hypernatremia- OR: 2.5, 95\% CI: 2.08-3.04, $P<0.001)$. Furthermore, hyponatremia on admission was associated with higher body temperature on admission $\left(\geq 38^{\circ} \mathrm{C}\right.$ ) (OR: 2.7, 95\% CI: 1.86-3.85, $P<0.001$ ) compared to patients with normonatremia, whereas hypernatremia showed no difference compared to normonatremia $(P=0.98)$.

Clinical symptoms of subgroups on admission are shown in Table 3. Patients with COVID-19 reported significant more often fever on admission as compared to controls (OR: 1.6, 95\% CI: 1.14-2.25, $P=0.005$ ). Irrespective of the COVID-19 status, hyponatremia as compared to normonatremia on admission was associated with symptoms such as fever (OR: 2.1, 95\% CI: 1.55-2.97, $P<0.001$ ) and shivering (OR: 1.95, 95\% CI: 1.33-2.86, $P<0.001)$. Hypernatremia on admission showed no difference as compared to normonatremia in both groups, patients with COVID-19 and controls, regarding the described symptoms.

\section{Discussion}

Our study has four main findings. First, the prevalence of hyponatremia on admission is significantly higher 


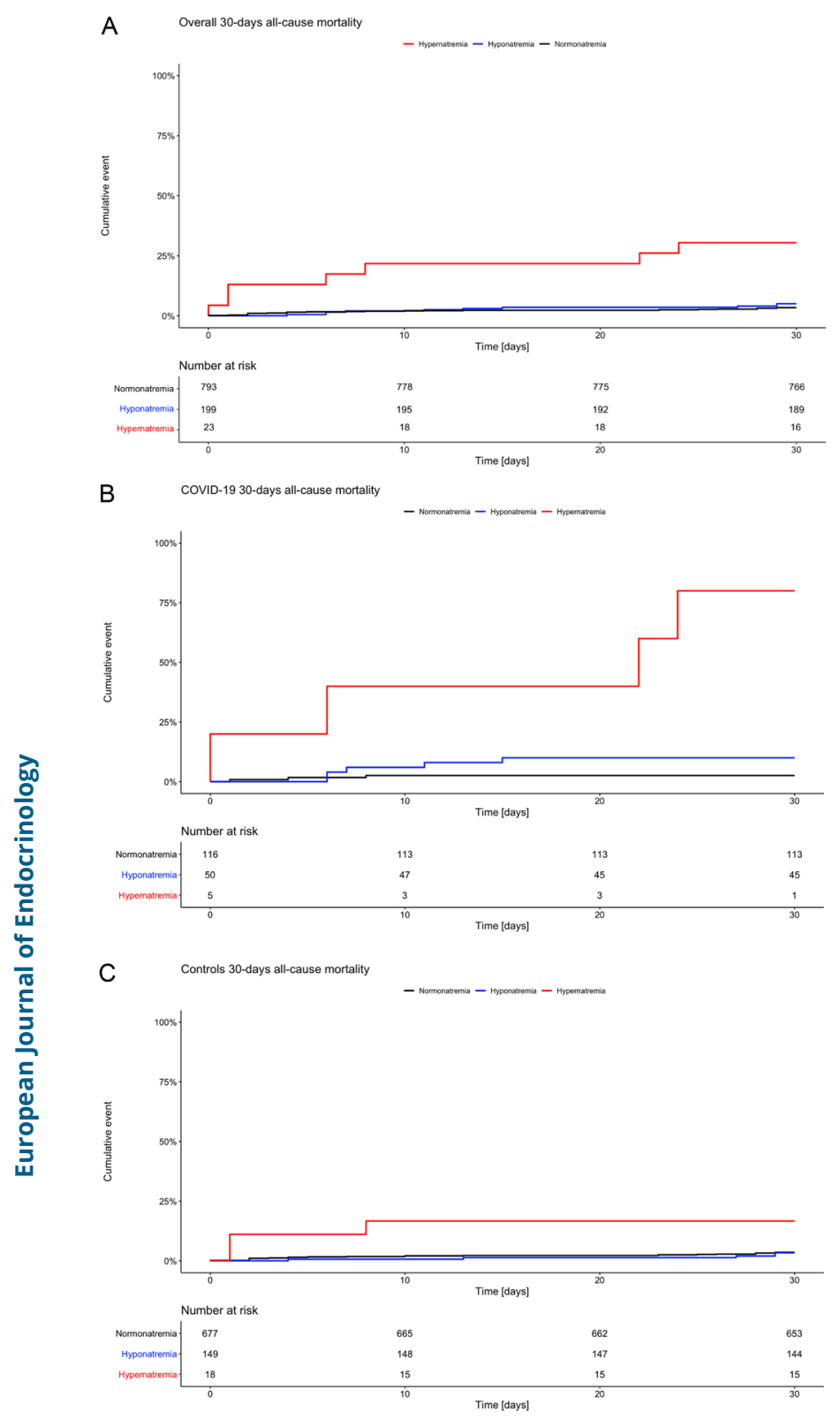

Figure 3

(A) Time to death graph of all patients. Kaplan-Meier curves displaying probability to death within the first 30 days after admission for all patients in each subgroup (hyponatremia, normonatremia, and hypernatremia) $(n=1015)$. Patients with hypernatremia (red) have had an increased risk of death compared to normonatremic patients with a hazard ratio of 2.1. (B) Time to death graph in COVID-19. Kaplan-Meier curves displaying probability to death within the first 30 days after admission in COVID-19 in each subgroup (hyponatremia, normonatremia, and hypernatremia) $(n=171)$. Patients with dysnatremia have had an increased risk of death compared to normonatremic patients, with a hazard ratio of 11.5 for in patients with COVID-19 in comparison to controls. Secondly, hyponatremia in COVID-19 but not in controls showed a significant association to 30-day mortality in comparison to normonatremia. Thirdly, hypernatremia on admission was associated with 30-day mortality, in both, patients with and without COVID-19. Forthly, hyponatremia and hypernatremia on admission were associated with adverse outcome and severity parameters in both groups.

The prevalence of hyponatremia and hypernatremia in our study is similar to previously published data of a large cohort with COVID-19, where the incidence of hyponatremia was 30\%, whilst hypernatremia was seen with a prevalence of $4 \%$ (22). Our study, however, in contrast to previous studies, includes a control group with patients without COVID-19 but similar symptoms, and we here show for the first time that patients with COVID19 experience a significantly higher rate of hyponatremia compared to controls. In addition, we could confirm the association between hyponatremia and mortality in COVID-19 $(22,23)$, moreover, we show for the first time that this association is significantly higher in comparison to a control group. Different mechanisms leading to an increased prevalence of hyponatremia in pneumonia have been postulated for example, the syndrome of inappropriate antidiuresis (SIAD) $(24,25)$, drug-induced hyponatremia (mainly diuretics or psychotropics), and microbial-induced hyponatremia (Legionella pneumonia) (26). These mechanisms could also be linked to the unfavorable outcome. One hypothesis is that the immune system may lead to the development of hyponatremia in patients with pneumonia and more specifically in patients with COVID-19 (27). In a recent meta-analysis of COVID-19 patients, a high rate of increased IL-1 and IL-6 was observed (28). IL-6 is presumed to play a role in sodium homeostasis by non-osmotically releasing arginine vasopressin (AVP) $(29,30)$. In support of this hypothesis, Berni et al. showed in a small cohort of 29 patients with COVID-19 that hyponatremia improved after anti-IL-6 therapy (31). In our study, we see

hypernatremia (red) and with a hazard ratio of 1.4 for hyponatremia (blue). (C) Time to death graph in controls. Kaplan-Meier curves displaying probability to death within the first 30 days after admission in controls in each subgroup (hyponatremia, normonatremia, and hypernatremia) $(n=844)$. Patients with hypernatremia (red) have had an increased risk of death compared to normonatremic patients with a hazard ratio of 5.3 . 
Table 2 Follow-up and outcome data. Data are presented as $n$ (\%) and median (IQR: 25th-75th percentile).

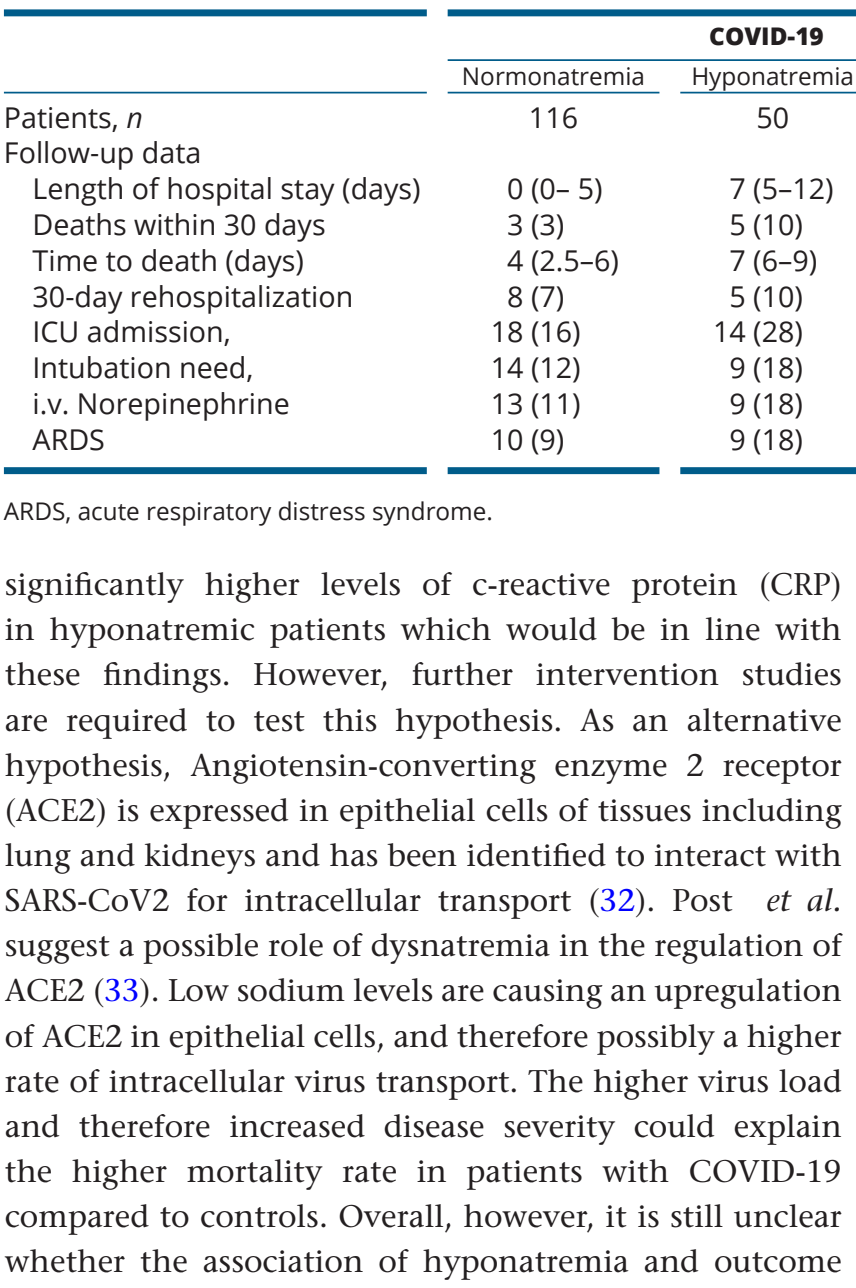

mirrors causality or simply defines hyponatremia as a marker for severity of disease.

Hypernatremia was also associated with a higher mortality rate in our study, as shown previously for patients with pneumonia in general (34). Hypernatremia is often a reflection of water loss rather than sodium gain, mostly due to dehydration due to increased body temperature (35). Fever was one of the most common symptoms in our analysis and could therefore partly explain the high rate of hypernatremia. Another common cause is drug-induced dehydration, in particular overuse of diuretics (36). Interestingly, our data show the highest rate of diuretics in hypernatremic patients in both groups. Diuretics in addition to age-related physiologic changes such as decreased thirst drive can lead to reduced net body water (37). Furthermore, studies in critical ill patients

Table 3 Clinical characteristics of patients with normonatremia, hyponatremia and hypernatremia on admission. Data are presented as $n$ (\%) and median (IQR: 25th -75th percentile).

\begin{tabular}{|c|c|c|c|c|c|c|}
\hline & \multicolumn{3}{|c|}{ COVID-19 } & \multicolumn{3}{|c|}{ Controls } \\
\hline & Normonatremia & Hyponatremia & Hypernatremia & Normonatremia & Hyponatremia & Hypernatremia \\
\hline Patients, $n$ & 117 & 50 & 5 & 682 & 149 & 18 \\
\hline \multicolumn{7}{|l|}{ Symptoms on admission } \\
\hline Fever & $59(51)$ & $29(58)$ & $2(40)$ & $243(36)$ & $81(54)$ & $6(33)$ \\
\hline Shivering & 22 (19) & $8(16)$ & $0(0)$ & $112(17)$ & $43(29)$ & $2(11)$ \\
\hline Cough & $79(68)$ & $28(56)$ & $3(60)$ & $360(53)$ & $72(48)$ & $9(50)$ \\
\hline Dyspnea & $51(44)$ & $20(40)$ & $2(40)$ & $357(53)$ & $54(36)$ & $8(44)$ \\
\hline Sore throat & $30(26)$ & $5(10)$ & $0(0)$ & $195(29)$ & $21(14)$ & $3(17)$ \\
\hline Diarrhea & 22 (19) & $14(28)$ & $0(0)$ & $99(15)$ & $25(17)$ & $2(11)$ \\
\hline Nausea/vomiting & $21(18)$ & $9(18)$ & $0(0)$ & $136(20)$ & $35(23)$ & $3(17)$ \\
\hline \multicolumn{7}{|l|}{ Clinical parameters } \\
\hline Temperature $\left({ }^{\circ} \mathrm{C}\right)$ & $37.1(36.6-37.8)$ & $37.4(36.9-38.5)$ & 36.7 (36.5-36.8) & $36.9(36.5-37.4)$ & $37.6(36.8-38.3)$ & $36.8(36.5-37.4)$ \\
\hline Respiratory rate (/min) & $20(16-23)$ & $23(17-25)$ & $26(24-26)$ & $18(16-22)$ & $20(16-24)$ & $21(16-27)$ \\
\hline $\mathrm{O}_{2}$ Saturation (\%) & $97(95-98)$ & 95 (93-97) & $95(89-96)$ & $97(96-98)$ & $96(95-98)$ & $96(93-98)$ \\
\hline Heart rate (/min) & $88(80-102)$ & $90(82-104)$ & $99(89-104)$ & $86(74-100)$ & $94(82-108)$ & $102(80-115)$ \\
\hline $\mathrm{SBP}(\mathrm{mmHg})$ & $136(125-151)$ & 133 (120-152) & $102(95-108)$ & $138(123-157)$ & $131(115-151)$ & 141 (122-167) \\
\hline $\mathrm{DBP}(\mathrm{mmHg})$ & $84(74,90)$ & $80(70,88)$ & $56(54-68)$ & $81(72-90)$ & $80(71-86)$ & 85 (77-96) \\
\hline $\mathrm{O}_{2}$-Therapy & $15(13)$ & $14(28)$ & $5(100)$ & $79(12)$ & $25(17)$ & $9(50)$ \\
\hline NEWS Total, $n$ (IQR) & $2(0-4)$ & $4(2-5)$ & $8(7-8)$ & $2(0-4)$ & $3(1-5)$ & $5(3-9)$ \\
\hline
\end{tabular}


showed high rates of dehydration caused by insensible water loss due to increased respiratory rate (38). Among all subgroups in our cohort, hypernatremic patients in COVID-19 and controls had the highest respiratory rates, which may have contributed to hypernatremia.

Previous studies showed increased rates of rehospitalization in hyponatremic and hypernatremic patients with pneumonia $(4,7)$. Interestingly, our study showed no link between hyponatremia or hypernatremia to 30-days rehospitalization in both, COVID-19 and controls, compared to normonatremia. However, our data on mortality indicated an unfavorable outcome in patients with COVID-19 and dysnatremia. Our sample might be too small to find such an association, which is supported as the 30-day rehospitalization rate in our cohort was lower as compared to other publications (39).

Both hyponatremia and hypernatremia as compared to normonatremia were linked to other adverse patients' outcome. Especially hyponatremia is an established and recognized criterion in severity scores for pneumonia, for example, the Pneumonia Severity Index (PSI) (15). Interestingly, a recent analysis showed a direct relation of hyponatremia and low $\mathrm{PaO}_{2} / \mathrm{FiO}_{2}$ ratio, an important index for respiratory performance (31). Respiratory failure was the leading cause of death in our analysis. Accordingly, symptoms and parameters representing respiratory failure such as low oxygen level on admission, fever, and mechanical ventilation were significantly linked to hyponatremia as compared to normonatremia in both groups.

There are some limitations to this study. First, we have only limited information about the etiology of dysnatremia, neither about psychotropic nor diuretic medication that could lead to drug induced SIAD. Other studies have investigated this question suggesting SIAD or hypovolemia to be main causes for dysnatremia. Due to the lack of information regarding the clinical volaemic status and further laboratory parameters, a reliable diagnosis of the etiology of hyponatremia was not possible.

Secondly, the study was insufficiently powered for some secondary analyses such as rehospitalization.

A major strength of our study is the comparison to a control group with suspected COVID-19 and thus similar symptoms, but a negative nasopharyngeal swab test for COVID-19. To the best of our knowledge, this is the first study with a control group and thus adds valuable information to the literature.

In conclusion, our data show that patients with COVID19 presented significantly more often with hyponatremia on admission compared to controls. Hyponatremia and hypernatremia in COVID-19 is associated with increased mortality. This underlines the importance of considering dysnatremia as a predictive outcome marker in COVID-19.

\section{Declaration of interest}

R Twerenbold reports research support from the Swiss National Science Foundation (Grant No P300PB_167803), the Swiss Heart Foundation, the Swiss Society of Cardiology, the Cardiovascular Research Foundation Basel, the University of Basel and the University Hospital Basel and speaker honoraria/consulting honoraria from Abbott, Amgen, Astra Zeneca, Roche, Siemens, Singulex and Thermo Scientific BRAHMS.

\section{Funding}

The COVIVA study was supported by the Swiss Heart Foundation and the Cardiovascular Research Foundation Basel.

\section{Author contribution statement}

C Atila and C O Sailer have contributed equally and share first authorship. R Twerenbold and M Christ-Crain have contributed equally and share senior authorship.

\section{Acknowledgements}

The authors thank the clinical staff for their valuable help during the study conduction. We thank all participants for their participation in the study.

\section{References}

1 Wang D, Hu B, Hu C, Zhu F, Liu X, Zhang J, Wang B, Xiang H, Cheng Z, Xiong Y, et al. Clinical characteristics of 138 hospitalized patients with 2019 novel coronavirus-infected pneumonia in Wuhan, China. JAMA 2020323 1061-1069. (https://doi.org/10.1001/ jama.2020.1585)

2 Guan WJ, Ni ZY, Hu Y, Liang WH, Ou CQ, He JX, Liu L, Shan H, Lei CL, Hui DSC, et al. Clinical characteristics of coronavirus disease 2019 in China. New England Journal of Medicine 2020382 1708-1720. (https://doi.org/10.1056/NEJMoa2002032)

3 Qian GQ, Yang NB, Ding F, Ma AHY, Wang ZY, Shen YF, Shi CW, Lian X, Chu JG, Chen L, et al. Epidemiologic and clinical characteristics of 91 hospitalized patients with COVID-19 in Zhejiang, China: a retrospective, multi-centre case series. QJM 2020 113 474-481. (https://doi.org/10.1093/qjmed/hcaa089)

4 Krüger S, Ewig S, Giersdorf S, Hartmann O, Frechen D, Rohde G, Suttorp N, Welte T \& CAPNETZ study group. Dysnatremia, vasopressin, atrial natriuretic peptide and mortality in patients with community-acquired pneumonia: results from the german competence network CAPNETZ. Respiratory Medicine 2014108 1696-1705. (https://doi.org/10.1016/j.rmed.2014.09.014)

5 Corrado RE, Lee D, Lucero DE, Varma JK \& Vora NM. Burden of adult community-acquired, health-care-associated, hospital-acquired, and ventilator-associated pneumonia: New York City, 2010 to 2014. Chest 2017152 930-942. (https://doi.org/10.1016/j.chest.2017.04.162) 6 Cuesta M, Slattery D, Goulden EL, Gupta S, Tatro E, Sherlock M, Tormey W, O'Neill S \& Thompson CJ. Hyponatraemia in patients with community-acquired pneumonia; prevalence and aetiology, 
and natural history of SIAD. Clinical Endocrinology 201990 744-752. (https://doi.org/10.1111/cen.13937)

7 Nair V, Niederman MS, Masani N \& Fishbane S. Hyponatremia in community-acquired pneumonia. American Journal of Nephrology 200727 184-190. (https://doi.org/10.1159/000100866)

8 Schuetz P, Haubitz S, Christ-Crain M, Albrich WC, Zimmerli W, Mueller B \& ProHOSP Study Group. Hyponatremia and anti-diuretic hormone in Legionnaires' disease. BMC Infectious Diseases 201313 585. (https://doi.org/10.1186/1471-2334-13-585)

9 Upadhyay A, Jaber BL \& Madias NE. Incidence and prevalence of hyponatremia. American Journal of Medicine 2006 119(Supplement 1) S30-S35. (https://doi.org/10.1016/j.amjmed.2006.05.005)

10 Hoorn EJ, Lindemans J \& Zietse R. Development of severe hyponatraemia in hospitalized patients: treatment-related risk factors and inadequate management. Nephrology, Dialysis, Transplantation 200621 70-76. (https://doi.org/10.1093/ndt/gfi082)

11 Corona G, Giuliani C, Verbalis JG, Forti G, Maggi M \& Peri A. Hyponatremia improvement is associated with a reduced risk of mortality: evidence from a meta-analysis. PLOS ONE $2015 \mathbf{1 0}$ e0124105. (https://doi.org/10.1371/journal.pone.0124105)

12 Borroni G, Maggi A, Sangiovanni A, Cazzaniga M \& Salerno F. Clinical relevance of hyponatraemia for the hospital outcome of cirrhotic patients. Digestive and Liver Disease 200032 605-610. (https://doi.org/10.1016/s1590-8658(00)80844-0)

13 Palevsky PM, Bhagrath R \& Greenberg A. Hypernatremia in hospitalized patients. Annals of Internal Medicine 1996124 197-203. (https://doi.org/10.7326/0003-4819-124-2-199601150-00002)

14 Katan M, Muller B \& Christ-Crain M. Copeptin: a new and promising diagnostic and prognostic marker. Critical Care 200812 117. (https:// doi.org/10.1186/cc6799)

15 Fine MJ, Auble TE, Yealy DM, Hanusa BH, Weissfeld LA, Singer DE, Coley CM, Marrie TJ \& Kapoor WN. A prediction rule to identify low-risk patients with community-acquired pneumonia. New England Journal of Medicine 1997336 243-250. (https://doi.org/10.1056/ NEJM199701233360402)

16 Zilberberg MD, Exuzides A, Spalding J, Foreman A, Jones AG, Colby C \& Shorr AF. Hyponatremia and hospital outcomes among patients with pneumonia: a retrospective cohort study. $B M C$ Pulmonary Medicine 20088 16. (https://doi.org/10.1186/1471-2466-816)

17 Alshayeb HM, Showkat A, Babar F, Mangold T \& Wall BM. Severe hypernatremia correction rate and mortality in hospitalized patients. American Journal of the Medical Sciences 2011341 356-360. (https:// doi.org/10.1097/MAJ.0b013e31820a3a90)

18 O'Shea PM, Lee GR, Griffin TP, Tormey V, Hayat A, Costelloe SJ, Griffin DG, Srinivasan S, O'Kane M, Burke CM, et al. COVID-19 in adults: test menu for hospital blood science laboratories. Irish Journal of Medical Science 2020189 1147-1152. (https://doi.org/10.1007/ s11845-020-02252-0)

19 von Elm E, Altman DG, Egger M, Pocock SJ, Gøtzsche PC, Vandenbroucke JP \& STROBE Initiative. The strengthening the reporting of observational studies in epidemiology (STROBE) statement: guidelines for reporting observational studies. International Journal of Surgery 201412 1495-1499. (https://doi. org/10.1016/j.ijsu.2014.07.013)

20 Smith GB, Redfern OC, Pimentel MA, Gerry S, Collins GS, Malycha J, Prytherch D, Schmidt PE \& Watkinson PJ. The national early warning score 2 (NEWS2). Clinical Medicine 201919 260. (https://doi. org/10.7861/clinmedicine.19-3-260)

21 R Core T. R: A Language and Environment for Statistical Computing 2018.

22 Frontera JA, Valdes E, Huang J, Lewis A, Lord AS, Zhou T, Kahn DE, Melmed K, Czeisler BM, Yaghi S, et al. Prevalence and impact of hyponatremia in patients with coronavirus disease 2019 in New York City. Critical Care Medicine 202048 e1211-e1217. (https://doi. org/10.1097/CCM.0000000000004605)
23 Tezcan ME, Dogan Gokce G, Sen N, Zorlutuna Kaymak N \& Ozer RS. Baseline electrolyte abnormalities would be related to poor prognosis in hospitalized coronavirus disease 2019 patients. New Microbes and New Infections 202037 100753. (https://doi.org/10.1016/j. nmni.2020.100753)

24 Spasovski G, Vanholder R, Allolio B, Annane D, Ball S, Bichet D, Decaux G, Fenske W, Hoorn EJ, Ichai C, et al. Clinical practice guideline on diagnosis and treatment of hyponatraemia. European Journal of Endocrinology 2014170 G1-G47. (https://doi.org/10.1530/ EJE-13-1020)

25 Verbalis JG, Goldsmith SR, Greenberg A, Korzelius C, Schrier RW, Sterns RH \& Thompson CJ. Diagnosis, evaluation, and treatment of hyponatremia: expert panel recommendations. American Journal of Medicine 2013 126(Supplement 1) S1-S42. (https://doi.org/10.1016/j. amjmed.2013.07.006)

26 Fiumefreddo R, Zaborsky R, Haeuptle J, Christ-Crain M, Trampuz A, Steffen I, Frei R, Müller B \& Schuetz P. Clinical predictors for Legionella in patients presenting with community-acquired pneumonia to the emergency department. BMC Pulmonary Medicine 20099 4. (https://doi.org/10.1186/1471-2466-9-4)

27 Ruscitti P, Berardicurti O, Iagnocco A \& Giacomelli R. Cytokine storm syndrome in severe COVID-19. Autoimmunity Reviews 202019 102562. (https://doi.org/10.1016/j.autrev.2020.102562)

28 Henry BM, Oliveira MHS de, Benoit S, Plebani M \& Lippi G. Hematologic, biochemical and immune biomarker abnormalities associated with severe illness and mortality in coronavirus disease 2019 (COVID-19): a meta-analysis. Clinical Chemistry and Laboratory Medicine 202058 1021-1028. (https://doi.org/10.1515/cclm-20200369)

29 Hodax JK, Bialo SR \& Yalcindag A. SIADH in systemic JIA resolving after treatment with an IL-6 inhibitor. Pediatrics 2018 141. (https:// doi.org/10.1542/peds.2016-4174)

30 Yousaf Z, Al-Shokri SD, Al-Soub H \& Mohamed MFH. COVID-19associated SIADH: a clue in the times of pandemic! American Journal of Physiology. Endocrinology and Metabolism 2020318 E882-E885. (https://doi.org/10.1152/ajpendo.00178.2020)

31 Berni A, Malandrino D, Parenti G, Maggi M, Poggesi L \& Peri A. Hyponatremia, IL-6, and SARS-CoV-2 (COVID-19) infection: may all fit together? Journal of Endocrinological Investigation 202043 1137-1139. (https://doi.org/10.1007/s40618-020-01301-w)

32 Chen L, Li X, Chen M, Feng Y \& Xiong C. The ACE2 expression in human heart indicates new potential mechanism of heart injury among patients infected with SARS-CoV-2. Cardiovascular Research 2020116 1097-1100. (https://doi.org/10.1093/cvr/ cvaa078)

33 Post A, Dullaart RPF \& Bakker SJL. Sodium status and kidney involvement during COVID-19 infection. Virus Research 2020286 198034. (https://doi.org/10.1016/j.virusres.2020.198034)

$34 \mathrm{Hu}$ J, Wang Y, Geng X, Chen R, Zhang P, Lin J, Teng J, Zhang X \& Ding X. Dysnatremia is an independent indicator of mortality in hospitalized patients. Medical Science Monitor 201723 2408-2425. (https://doi.org/10.12659/msm.902032)

35 Nigro N, Winzeler B, Suter-Widmer I, Schuetz P, Arici B, Bally M, Refardt J, Betz M, Gashi G, Urwyler SA, et al. Copeptin levels and commonly used laboratory parameters in hospitalised patients with severe hypernatraemia-the \&quot;Co-MED study\&quot. Critical Care 201822 33. (https://doi.org/10.1186/s13054-018-1955-7)

36 Oude Lansink-Hartgring A, Hessels L, Weigel J, Smet AMGA de, Gommers D, Panday PVN, Hoorn EJ \& Nijsten MW. Longterm changes in dysnatremia incidence in the ICU: a shift from hyponatremia to hypernatremia. Annals of Intensive Care 2016622. (https://doi.org/10.1186/s13613-016-0124-x).

37 Morley JE. Dehydration, hypernatremia, and hyponatremia. Clinics in Geriatric Medicine 201531 389-399. (https://doi.org/10.1016/j. cger.2015.04.007) 
38 Li Li C, Oi Yan T, Ming Chit Arthur K, Hoi Ping S, King Chung Kenny C $\&$ Wing Wa Y. Insensible water loss through adult extracorporeal membrane oxygenation circuit: an in vitro study. ASAIO Journal 201460 508-512. (https://doi.org/10.1097/MAT.0000000000000098)
39 Chawla A, Sterns RH, Nigwekar SU \& Cappuccio JD. Mortality and serum sodium: do patients die from or with hyponatremia? Clinical Journal of the American Society of Nephrology 20116 960-965. (https:// doi.org/10.2215/CJN.10101110)

Received 30 November 2020

Revised version received 6 January 2021

Accepted 12 January 2021 